\title{
PnB Designer: a web application to design prime and base editor guide RNAs for animals and plants
}

\author{
Sebastian M. Siegner ${ }^{1}$, Mehmet E. Karasu', Markus S. Schröder ${ }^{1}$, Zacharias Kontarakis ${ }^{1,2}$ and Jacob E. Corn ${ }^{1 *}$ (i)
}

\author{
${ }^{*}$ Correspondence: \\ jacob.corn@biol.ethz.ch \\ 1 Department of Biology, ETH \\ Zurich, Zurich, Switzerland \\ Full list of author information \\ is available at the end of the \\ article
}

\begin{abstract}
Background: The rapid expansion of the CRISPR toolbox through tagging effector domains to either enzymatically inactive Cas9 (dCas9) or Cas9 nickase (nCas9) has led to several promising new gene editing strategies. Recent additions include CRISPR cytosine or adenine base editors (CBEs and ABEs) and the CRISPR prime editors (PEs), in which a deaminase or reverse transcriptase are fused to $\mathrm{nCas9}$, respectively. These tools hold great promise to model and correct disease-causing mutations in animal and plant models. But so far, no widely-available tools exist to automate the design of both $\mathrm{BE}$ and PE reagents.
\end{abstract}

Results: We developed PnB Designer, a web-based application for the design of pegRNAs for PEs and guide RNAs for BEs. PnB Designer makes it easy to design targeting guide RNAs for single or multiple targets on a variant or reference genome from organisms spanning multiple kingdoms. With PnB Designer, we designed pegRNAs to model all known disease causing mutations available in ClinVar. Additionally, PnB Designer can be used to design guide RNAs to install or revert a SNV, scanning the genome with one $C B E$ and seven different $A B E$ PAM variants and returning the best $B E$ to use. PnB Designer is publicly accessible at http://fgcz-shiny.uzh.ch/PnBDesigner/

Conclusion: With PnB Designer we created a user-friendly design tool for CRISPR PE and $\mathrm{BE}$ reagents, which should simplify choosing editing strategy and avoiding design complications.

Keywords: Prime editing, Base editing, Guide RNA design, Web application

\section{Background}

The landscape of genome engineering has changed drastically since the discovery of the CRISPR (clustered regularly interspaced short palindromic repeats) locus and the associated Cas9 protein [1]. After the successful application of gene editing in human cells, CRISPR-Cas9 research created a totally new and fast-moving field with an exploding number of publications $[1,2]$. Directed by a guide RNA (gRNA), the endonuclease activity of Cas9 protein introduces a double strand break (DSB) at the target locus [1]. Each Cas9-induced DSB can be resolved as an error-prone insertion or deletion (indel) author(s) and the source, provide a link to the Creative Commons licence, and indicate if changes were made. The images or other third party material in this article are included in the article's Creative Commons licence, unless indicated otherwise in a credit line to the material. If material is not included in the article's Creative Commons licence and your intended use is not permitted by statutory regulation or exceeds the permitted use, you will need to obtain permission directly from the copyright holder. To view a copy of this licence, visit http:// creativecommons.org/licenses/by/4.0/. The Creative Commons Public Domain Dedication waiver (http://creativecommons.org/publi cdomain/zero/1.0/) applies to the data made available in this article, unless otherwise stated in a credit line to the data. 
or a precise, templated homology directed repair (HDR) event [3]. Fusing various effector domains to catalytically impaired versions of Cas proteins has led to an explosion of tools with outcomes beyond indels and HDR [2].

CRISPR base editors (BEs) use a cytidine- or adenine-deaminase protein to generate a DNA base transitions from $\mathrm{C} \rightarrow \mathrm{T}$ or $\mathrm{A} \rightarrow \mathrm{G}$, respectively $[4,5]$. While the gRNA bound to the Cas enzyme directs the $\mathrm{BE}$ to a genomic locus, the deaminase can edit its target base within a certain window $[4,5]$. Base editors have been used in many animal and plant models and hold a great promise for the correction of disease-causing single nucleotide variants (SNVs) in a broad spectrum of diseases [6-11]. Adenine-deaminase base editors (ABEs) in particular exhibit a promising combination of a high efficiency at the on-target site with low off-target DNA and RNA editing [12, 13]. In contrast, some of the early CBEs versions suffered from off-target activity [12], and only the newly engineered versions of these CBEs or Target-AID based CBEs exhibit sufficient specificity to be considered for therapeutic applications [13-15].

Even more recently, Prime Editors (PEs) have introduced the possibility of small programmable genomic changes without HDR. The PE2 consists of a Cas9 nickase fused to an engineered reverse transcriptase [16]. To introduce a modification in the genome, PEs use a prime editing extended guide RNA (pegRNA), consisting of a 20 nt guide sequence, a primer binding site (PBS) and a reverse transcriptase template (RTT) [16]. The guide directs the Cas enzyme to a target site, the PBS hybridizes to the opposite strand to prime the reverse transcriptase, and the RTT integrates the desired genomic alteration. To further optimize PE2, the PE3 system uses an additional guide that directs a nick to the non-edited complementary strand, shifting the equilibrium of DNA repair to favor the edited strand [16]. This secondary nicking can lead to indels if the edited strand is nicked before the RTT sequence has been incorporated, which was recently shown to be the case [17]. However, the PE3b system solves this problem by using a second guide against the anticipated edit, such that the nick on the wild-type strand only occurs after successful sequence incorporation on the edited strand [16]. Therefore, for users who would like to avoid potential off targets, we recommend using either the PE2 or PE3b system.

The manual design of a complex pegRNA and multiple nicking sites can be laborious, challenging and error-prone, especially in these early days of prime editing when optimization may require the design and testing of several pegRNAs. Software to easily design pegRNAs would make this exciting new technology more accessible to a wide user base. Several web-based tools exist for the design of BEs [18-20]. However, to the best of our knowledge there is no publicly available software that can design both base editing and prime editing gRNAs.

Here, we present the PnB Designer tool, a web-based application for gRNA design for PE, CBE, and the most recent ABEs such as ABEmax and ABE8e [21, 22]. PnB Designer provides the user with an intuitive interface to make editing strategy and target site selection straightforward. It generates helpful output commands to lead the user through design problems and presents the resulting guides in a simple and easy to understand fashion. We have also used PnB Designer to design candidate pegRNAs to model all human mutations in ClinVar. Multiple types of ClinVar-targeting pegRNA designs are included to assist in the design of experiments using PEs. PnB Designer 
compares favorably to other $\mathrm{BE}$ design webservers and is the first $\mathrm{BE}$ and $\mathrm{PE}$ design webserver of which we are aware (Table 1).

\section{Implementation}

PnB Designer is a web application to design gRNAs for CRISPR prime and base editors. Written in R, PnB Designer is constructed with the Shiny package [23] and Bioconductor $[24,25]$. The application includes feedback after each input to help users avoid flaws in the complex design of base and prime editing gRNAs. The user-friendly interface combined with instructions and explanatory pop-up windows in the result visualization make it accessible to users on all levels.

\begin{tabular}{|c|c|c|c|c|c|c|}
\hline Tool & $\begin{array}{l}\text { PnB } \\
\text { Designer }\end{array}$ & SNP-CRISPR & BE-FF & beditor & BE-Designer & Benchling \\
\hline $\begin{array}{l}\text { pegRNA } \\
\text { design for } \\
\text { prime edit- } \\
\text { ing }\end{array}$ & $\checkmark$ & $\times$ & $\times$ & $\times$ & $x$ & $x$ \\
\hline $\begin{array}{l}\text { Protospacer } \\
\text { design for } \\
\text { prime edit- } \\
\text { ing }\end{array}$ & $\checkmark$ & $\checkmark$ & $\times$ & $x$ & $x$ & $\times$ \\
\hline $\begin{array}{l}\text { Integrated } \\
\text { PAM variants } \\
\text { for ABE }\end{array}$ & 7 & 0 & 6 & 6 & 6 & 0 \\
\hline $\begin{array}{l}\text { Assessment of } \\
\text { off-targets }\end{array}$ & $\times$ & $\checkmark$ & $\checkmark$ & $\checkmark$ & $\checkmark$ & $\checkmark$ \\
\hline $\begin{array}{l}\text { Support mul- } \\
\text { tiple sample } \\
\text { file import }\end{array}$ & $\checkmark$ & $\checkmark$ & $\times$ & $\checkmark$ & $\checkmark$ & $\times$ \\
\hline $\begin{array}{l}\text { Explanatory } \\
\text { feedback } \\
\text { after wrong } \\
\text { input }\end{array}$ & $\checkmark$ & $\checkmark$ & $x$ & $\checkmark$ & $\times$ & $\checkmark$ \\
\hline $\begin{array}{l}\text { Guide design } \\
\text { on non- } \\
\text { reference } \\
\text { genome }\end{array}$ & $\checkmark$ & $\checkmark$ & $\checkmark$ & $x$ & $x$ & $x$ \\
\hline Access & Webserver & Webserver & Webserver & $\begin{array}{l}\text { Installation } \\
\text { in python } \\
\text { environ- } \\
\text { ment and } \\
\text { GUI or com- } \\
\text { mand line }\end{array}$ & Webserver & $\begin{array}{l}\text { Integrated fea- } \\
\text { ture on the } \\
\text { benchling } \\
\text { website }\end{array}$ \\
\hline Input format & $\begin{array}{l}\text { Genomic } \\
\text { coordinates, } \\
\text { text format } \\
\text { sequence } \\
\text { input by } \\
\text { user }\end{array}$ & $\begin{array}{l}\text { Genomic } \\
\text { coordinates } \\
\text { file only }\end{array}$ & $\begin{array}{l}\text { SNP ID, } \\
\text { genomic } \\
\text { coordinates, } \\
\text { text format } \\
\text { sequence } \\
\text { input by } \\
\text { user }\end{array}$ & $\begin{array}{l}\text { Genomic } \\
\text { coordinates } \\
\text { file only }\end{array}$ & $\begin{array}{l}\text { Genomic } \\
\text { coordinates } \\
\text { file, text } \\
\text { format } \\
\text { sequence } \\
\text { input by the } \\
\text { user }\end{array}$ & $\begin{array}{l}\text { DNA sequence } \\
\text { on the } \\
\text { benchling } \\
\text { website }\end{array}$ \\
\hline URL & $\begin{array}{l}\text { fgcz-shiny. } \\
\text { uzh.ch/PnB- } \\
\text { Designer/ }\end{array}$ & $\begin{array}{l}\text { flyrnai.org/ } \\
\text { tools/ } \\
\text { snp_crispr }\end{array}$ & $\begin{array}{l}\text { danioffenlab. } \\
\text { com/be-ff }\end{array}$ & $\begin{array}{c}\text { pypi.org/ } \\
\text { project/ } \\
\text { beditor }\end{array}$ & $\begin{array}{l}\text { rgenome. } \\
\text { net/be- } \\
\text { designer/ }\end{array}$ & benchling.com \\
\hline Reference & - & $\begin{array}{l}\text { Chen } \\
\text { et al. [38] }\end{array}$ & $\begin{array}{r}\text { Rabinowitz } \\
\text { et al. [18] }\end{array}$ & $\begin{array}{l}\text { Dandage } \\
\text { et al. [19] }\end{array}$ & $\begin{array}{l}\text { Hwang et al. } \\
{[20]}\end{array}$ & - \\
\hline
\end{tabular}


In each gRNA design run, the user can choose an editing strategy and target genome (Fig. 1a). In the 'Genomes panel' users can select genomes of commonly used organisms such as Homo sapiens, Mus musculus and Danio rerio. Moreover, since genome manipulation applications such as prime and base editing have been successfully deployed in plants $[9,26,27]$, Oryza sativa (Asian rice), Arabidopsis thaliana (Thale cress) and Vitis vinifera (Common Grape Vine) are also included in the 'Genomes panel'. Users can also input their own target sequence by selecting 'None of the above' in the 'Genomes panel' and 'Sequence input' in the later panel, enabling design of gRNAs for non-model organisms or synthetic constructs.

Finally, the user can decide either to design BE or PE gRNAs for a single edit, or to automatically design multiple gRNAs using a comma-separated value (CSV) format file as input (Fig. 1b). After processing the user's inquiries, PnB Designer allows the user to save the results of a gRNA search by clicking one of the download buttons, located below the output table.

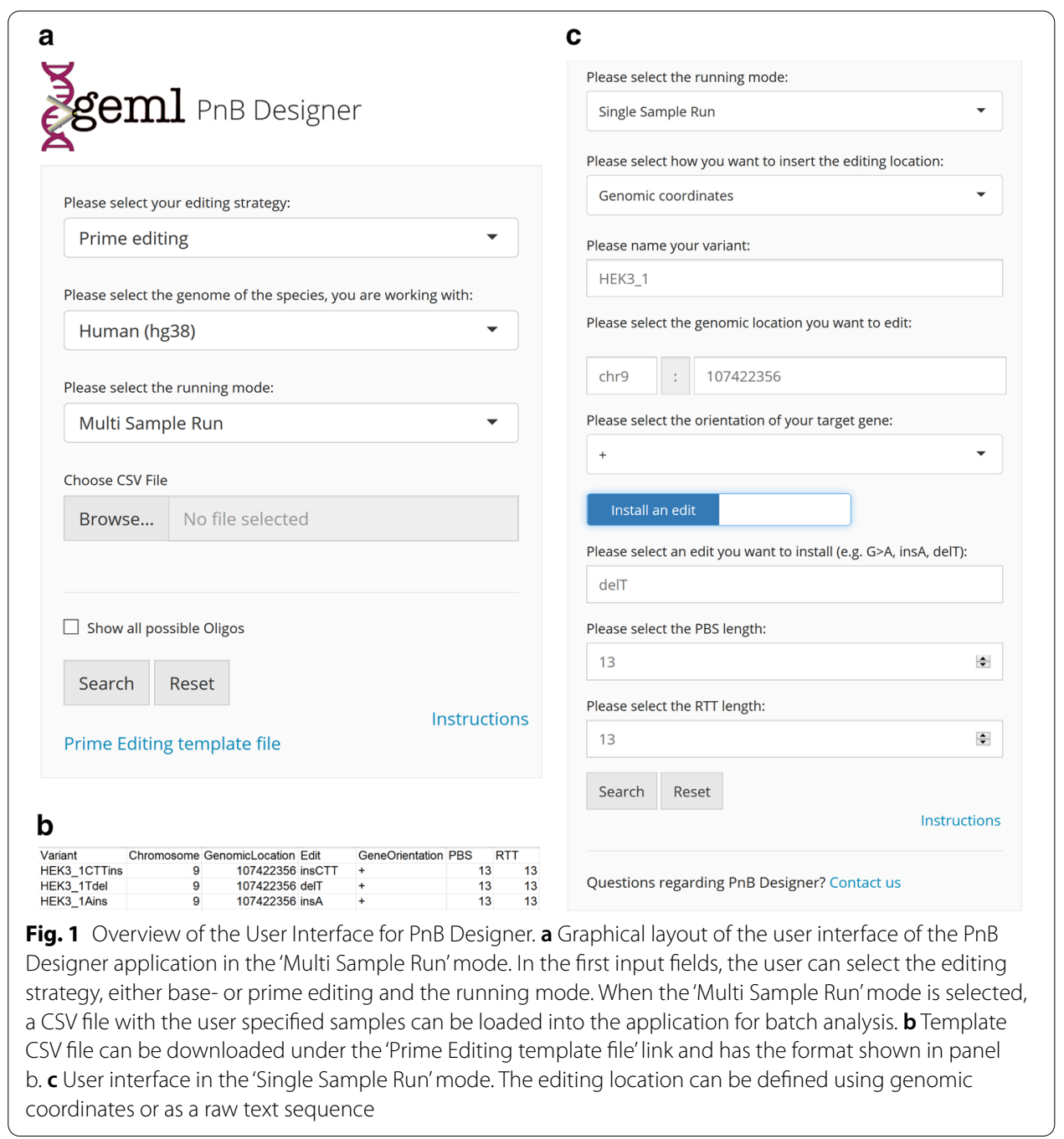




\section{Results}

\section{Prime editing with PnB Designer user interface}

Selecting the 'Prime editing' option, the user can install a desired edit or correct a certain mutation of any type (substitution, insertion, deletion) by setting the switch button to the left or right position (Fig. 1c). The user can use genomic coordinates or input a target sequence in text format (Fig. 1c). PBS and RTT length are important parameters for successful pegRNA design. While it is suggested to start with a PBS length of $\sim 13 \mathrm{nt}$ and RTT length of 10-16 nt, precise rules for their values have not yet been determined and pegRNA efficiency can be optimized by varying RTT length [16]. Hence, in PnB Designer, PBS and RTT lengths are by default set at suggested values of 13 nt but can be easily modified by the user.

\section{Design strategy for pegRNAs}

PnB Designer scans the sense and antisense strands to find all possible $5^{\prime}$-NGG-3 ${ }^{\prime}$ protospacer adjacent motif (PAM) sites around the edit position, beginning +6 nt to the $3^{\prime}$ end of the desired edit and then scanning $100 \mathrm{nt}$ in the $5^{\prime}$ direction, giving the user the option to choose also very distant PAMs. Non-NGG PAMs are currently not explored due to the lack of experimental validation with PEs, but expansion to other PAMs is anticipated and accordingly their support will be added to future versions of PnB Designer. All possible NGG PAMs are stored and evaluated in respect to their distance from the edit position and the input RTT length. A pegRNA is considered a possible candidate if the edit is fully covered by the RTT. PnB Designer then stores the protospacer, PBS, and RTT sequences. The intended edit on the coding strand is also highlighted for confirmation. Nicking guides for the PE3 and PE3b systems are designed and filtered to provide a suitable selection of gRNAs, according to recommendations by the Liu lab [16]. For PE3, only nicking guides 40-100 nt up/downstream of the initial nick are considered. For PE3b, only PAM sequences on the complementary strand that partially overlap with the PE2 PAM or protospacer sequence are displayed, as indicated in the PE3b manual design scheme suggested by the Liu lab.

\section{Prime editing with PnB Designer: result visualization}

After a successful run, the resulting sequences are shown in an output table (Fig. 2a, b) with the following parameters: the variant name, 'pegRNA Score', protospacer sequence, edit position relative to the PAM, PAM sequence, PAM strand and coding strand $3^{\prime}$ extension with implemented edits. As an indicator for the quality of each pegRNA, we implemented a 'pegRNA Score' equation based on the recommendations from the Liu lab [16]. Our intent was to automate several heuristic constraints that would otherwise need to be manually evaluated for each pegRNA, allowing the user to triage pegRNAs that are highly unlikely to work. The 'pegRNA Score' is thus similar to early scoring systems for normal gRNAs. As with DSB-forming Cas9 editing, large amounts of data on the efficiency of prime editing with many different pegRNAs may in future enable approaches such as machine learning to predict pegRNA efficiency.

The simple 'pegRNA Score' was implemented to make users aware of any design features of their pegRNA, which could impede the function of the pegRNA, when used 


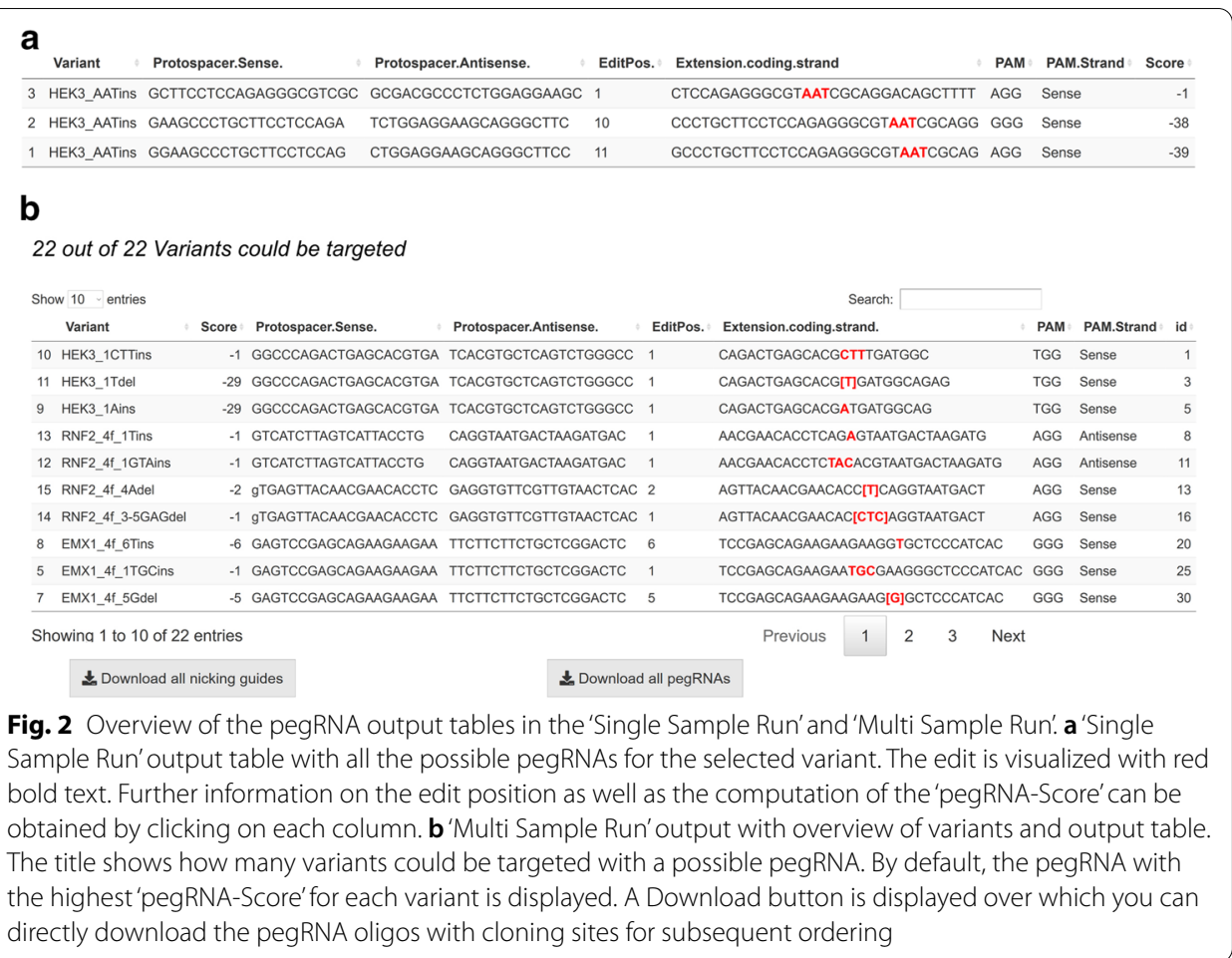

under the conditions described in Anzalone et al. [16]. The pegRNA Score follows the same penalty system as suggested by the Liu lab, with larger negative numbers are indicating worse pegRNA designs. In case of a $\mathrm{C}$ as the first base in the $3^{\prime}$ extension editing shows lower efficiency potentially due to disruption of gRNA structure by pairing with G81 of Cas9 [16] and a penalty score of -28 is given to this pegRNA, in this case increasing the RTT length is recommended. Multiple thymine (T) nucleotides (more than 4 ) in the $3^{\prime}$ extension of the pegRNA are strongly penalized (score -50 ), since the current pegRNA plasmid utilizes an RNA Polymerase III promoter and thymine stretches are recognized as transcription termination signals [28]. The influence of $\mathrm{T}$ stretches in a pegRNA was also found as one of the most important features, predicting a decrease in PE2 efficiency in one of the first prime editing screens [29]. Another important point to consider is the number of homologous bases after the intended edit. This should be more than $5 \mathrm{nt}$, or even more than $10 \mathrm{nt}$ if possible, to increase annealing at that end. Thus, a penalty score of -6 is assigned if the number of homologous nucleotides is less than five. Lastly, PE is most efficient if the edit is close to the initial nick site. Hence, making pegRNAs with an edit position within the protospacer or PAM is preferable (edit position 1-6) [16], and a penalty of - 1 per increase of the edit position is given to the pegRNA to show deviation from this optimal design. The sum of all penalties defines the 'pegRNA Score'. Examples of pegRNA designs with favorable (Additional file 1: Figure 1a, first row) and poor scores (Additional file 1: Figure 1a, last two rows) can be seen in the Supplementary information. PegRNAs that receive multiple penalties are highly unlikely to be active, and thus can be omitted from the testing. However, at this early stage of prime editing, we do not recommend using the pegRNA Score as a discriminator between similarly scoring pegRNAs. It is instead advisable for users to 
adopt loose thresholds and experimentally test multiple pegRNAs spanning a wide range of good scores. For example, all pegRNAs from Anzalone et al. Figure 4a have good pegRNA scores between -14 and -1 , but there is only a slight correlation between these reasonably-scoring pegRNAs and their efficiency. None of the pegRNAs with good editing efficiency reported by Anzalone et al. have a high pegRNA penalty, supporting the triage of pegRNAs with bad scores (Additional file 2: Figure 2).

From the output table, the user can select a desired pegRNA and, if they wish, additionally implement the PE3 or PE3b system. If so, they will be presented with potential nicking guides according to the design suggestions summarized above. After pegRNA and the optional nicking guide selection, their sequences can be directly downloaded including adapters for cloning into the BsmBI-digested acceptor vector from Anzalone et al. and the pegRNA acceptor vector deposited by the Liu lab on Addgene [\#132777].

In the output table of the multi sample mode only the pegRNA with the highest Score for a variant is shown. The user can also access all pegRNAs by selecting the 'Show all possible pegRNAs' box (Fig. 1a). For non-targetable variants with the specific input conditions, a row with possible reasons for the unsuccessful search is displayed. Additionally, users can download the oligonucleotides for all pegRNAs with cloning extensions for direct Golden Gate assembly into the pU6-pegRNA-GG-acceptor plasmid [16].

\section{Automated design of pegRNAs to reconstitute ClinVar genotypes using prime editing}

We used PnB Designer to design candidate pegRNAs to model all known human pathogenic or likely pathogenic nuclear-encoded variants in ClinVar [30] (https://ftp.ncbi.nlm. nih.gov/pub/clinvar/tab_delimited/variant_summary.txt.gz), filtered to target deletions, duplications, insertion and SNVs accessible to PE [16]. We started with an input ClinVar dataset containing 71,006 SNVs, 24,373 deletions, 10,199 duplications and 1677 insertions. We found that with a PBS and RTT length of 13 nt we could target 57,537 out of 71,006 SNVs (81\%), 17,471 of 24,373 (72\%) deletions, 7040 out of 10,199 (69\%) duplications and 1077 out of 1677 (64\%) insertions (Fig. 3a, b). Overall, we retrieved suitable pegRNA designs for $78 \%$ of the analyzed variants. Since the constraints on RTT length are not well defined experimentally, we repeated this analysis with RTT lengths ranging from 10 to $20 \mathrm{nt}$. As expected, fewer variants were targetable with a shorter RT template, and at the longest RTT length of $20 \mathrm{nt}$, we were able to design pegRNAs for $87 \%$ of all ClinVar input variants (Fig. 3c). With a RTT length of 13 nt the 'pegRNA Score' distribution shows that the majority of pegRNAs have a score of -20 and higher, which shows that only some pegRNAs suffer from additional penalties $\left(3^{\prime} \mathrm{C}\right.$ or multiple thymine in the extension) (Fig. 3d). The frequency of targetable variants did not yet plateau with a 20 nt RTT, but longer RTTs are likely to introduce problems in the already long pegRNA. In summary, we were able to successfully design gRNAs for prime editing for a large dataset of human variants using PnB Designer. All ClinVar-targeting pegRNAs of all RTT lengths can be accessed (Additional files).

\section{Base editing with PnB Designer: user interface}

When selecting the 'Base editing' option, the user can either input a target sequence in text format or select genomic coordinates, orientation of the target region and the SNV they want to revert to the reference wildtype sequence (Fig. 4a). The PnB Designer 


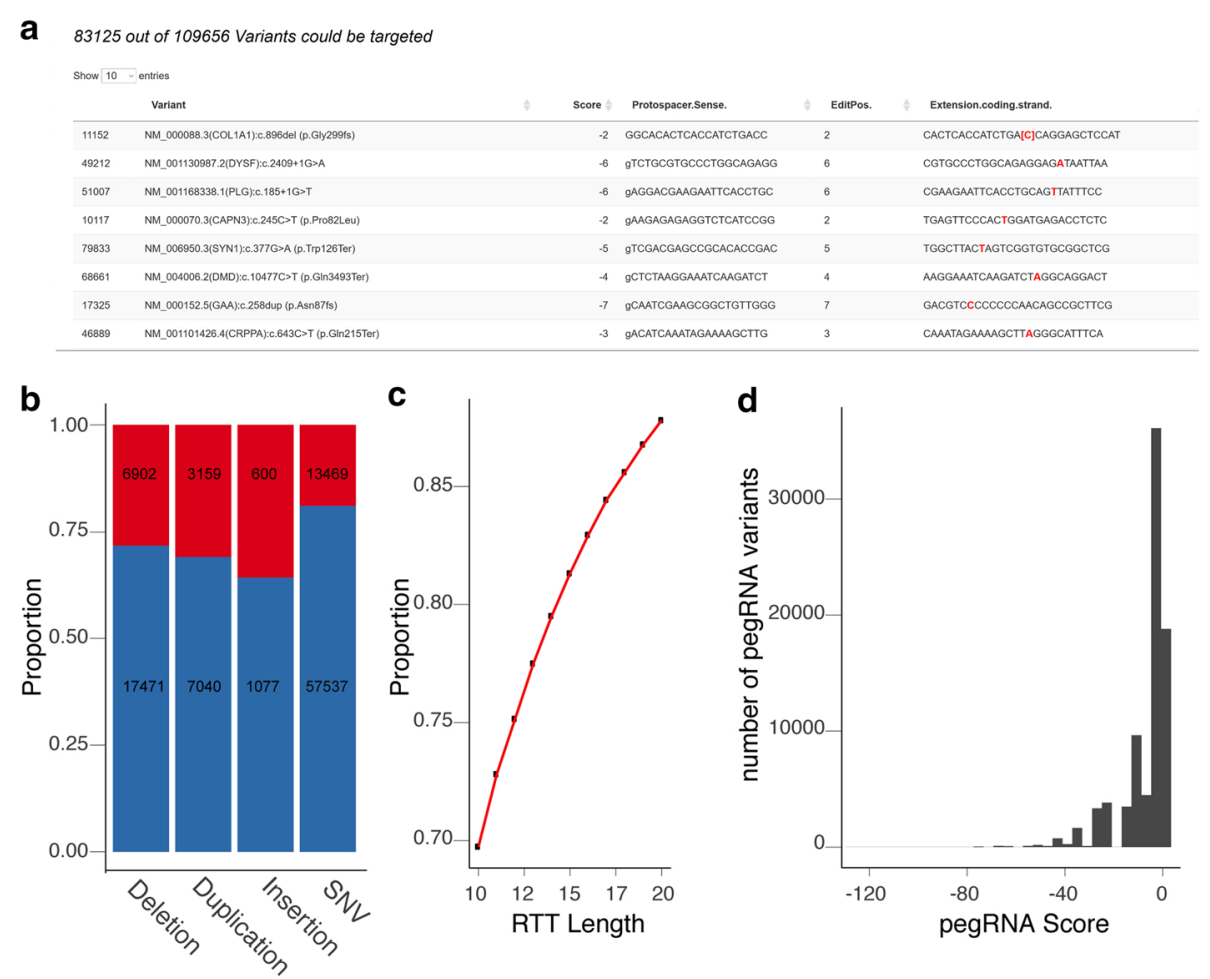

Fig. 3 pegRNA design for modelling all pathogenic and potentially pathogenic SNVs, insertions, duplications, and deletions in ClinVar. a Section of the output table after designing pegRNAs for a dataset of 109,565 variants. 83,125 variants were targetable using prime editing with a $13 \mathrm{nt}$ RTT. $\mathbf{b}$ Quantification of different type of variants in the dataset and proportion of variants targetable with PE for each type highlighted in blue, not targetable in red, respectively. The black number in the bars represent the number of variants (RTT length: 13 nt). c Proportion of pathogenic and likely pathogenic variants targetable using different lengths of RT templates (10-20 nt). d Distribution of the 'pegRNA-Score' of the highest possible score for each variant (RTT length: $13 \mathrm{nt})$. Most pegRNAs have a score greater than -20 , showing that these are possibly functional pegRNAs with no major design penalties. Figures were produced using the package ggplot2 [37] with R (Version 3.6.3)

supports gRNA design for ABEs and CBEs. Due to the known off-target propensities of early CBEs, only engineered versions with decreased off-targets have been implemented: BE3 (R33A), BE3 (R33A/K34A), BE3-hA3A (R128A) and Target-AID, all recognizing 5'-NGG-3' PAMs [13-15]. However, PnB Designer can design base editing gRNAs for ABEs based on SpCas9 (Streptococcus pyogenes) and SaCas9 (Streptococcus aureus) with a broad spectrum of PAM variants: 5'-NGG-3' (SpCas9), 5'-NGA-3' (SpCas9-VRQR), 5'-NGCG-3' (SpCas9-NG), 5'-NNGRRT-3' (SaCas9), 5'-NNNRRT-3' (SaCas9-KKH) and the newly by structure-guided engineering created versions SpG $5^{\prime}-\mathrm{NGN}-3^{\prime}$ and SpRY $5^{\prime}-\mathrm{NRN}-3^{\prime}$ and to a lesser extent $5^{\prime}-\mathrm{NYN}-3^{\prime}[5,31-35]$.

\section{Design strategy for BE gRNAs}

The user can either enter a sequence or genomic coordinates. During manual sequence input, the user can select the desired single base pair change (for example $A>G$ to convert an adenine at that position into a guanine). With the input of genomic coordinates, PnB Designer retrieves the genomic sequence from the selected reference genome and converts the specific variant sequence to include the SNV. The resulting sequence is 


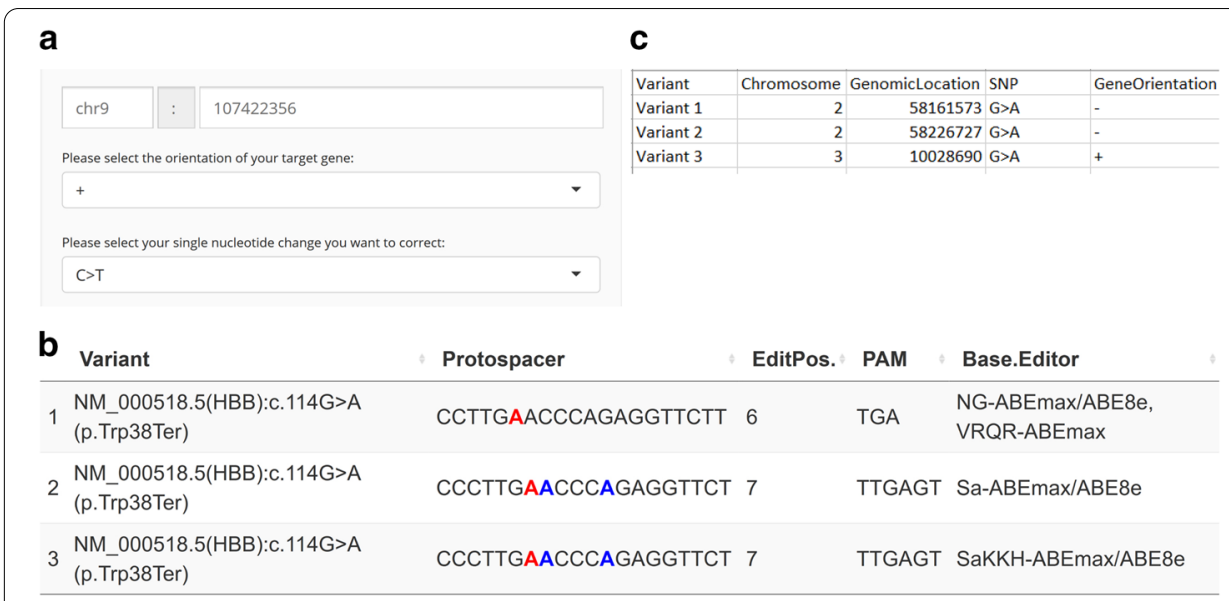

Fig. 4 Overview of base editing input and output. a Segment of the BE specific user interface. A target sequence in text format can be provided by the user to install an edit. For correcting a specific SNV, genomic coordinates and the orientation of the gene must be provided by the user. C $\rightarrow$ T or G $\rightarrow$ A SNVs can be selected for correction. $\mathbf{b}$ Example 'Single sample run' for a random ClinVar variant. The target adenine is highlighted in red. Other adenines in the editing window that could be modified are in blue. Edit position relative to the PAM, PAM sequence and the corresponding ABE are shown. $\mathbf{c}$ Example base editing template for the 'Multi sample run'

searched for PAM sites in the right distance to the SNV given the described editing windows of the Cas9-ABE and -CBE variants [5, 13-15, 31-35]. With ABEs, $\mathrm{C} \rightarrow \mathrm{T}$ genomic variants can be reverted by $\mathrm{A} \rightarrow \mathrm{G}$ conversion on the antisense strand to achieve the intended edit on the sense strand. Similarly, with CBEs, $\mathrm{C} \rightarrow \mathrm{T}$ and $\mathrm{G} \rightarrow \mathrm{A}$ conversions are possible. All previously described PAM variants with their respective editing window are tested against the edit, allowing the user to choose the best tool given the desired outcome. The editing windows are defined based on the experimental data. For example ABEmax was implemented with an editing window from base 5-7, with base 1 being the most distal from the PAM site [22]. Additionally for BE3 (R33A/K34A), the strong sequence preference for a $5^{\prime} \mathrm{T}$ next to the edit has been included as well [13].

\section{Base editing with PnB Designer: result visualization}

Resulting gRNAs are displayed in the output table, with the target base highlighted in red. Bystander adenines or cytosines in a given editing window that could also be modified are highlighted in blue, giving an indication about potential off-targets of the deaminase at the on-target site. With this information users can easily select the "cleanest" gRNAs (Fig. 4b). The output table consists of the 20 nt protospacer sequence (targeting the variant sequence), edit position, PAM site and suggested base editor that can target this variant. Additional information about the edit position can be found by clicking on the respective column. The newly developed near-PAMless SpCas9 variant SpRY base editor has lower overall efficiency when compared to the other implemented base editors [31]. We included this information in the output table to guide the user to the most efficient editing strategy when multiple ABEs are able to target a site. An example for a good base editing guide with no bystander bases can be seen in the Supplementary Information (Additional file 1: Figure 1b, third row). 


\section{Conclusion}

In summary, PnB Designer is a flexible and user-friendly web application for single and batch design (Figs. 1b, 4c) of PE pegRNAs and BE gRNAs. PnB Designer allows users to design pegRNAs with ease, lowering the barrier to entry for PE applications. Additionally, we implemented most recent CBEs and ABEs with a wide variety of PAM variants, which are not yet available in other base editing design webservers (Table 1). While other BE design tools provide custom base editor options to choose new PAMs and editing windows, we think implementation of the newest base editors with their experimentally established editing windows makes it more user friendly. As the base editor field is rapidly moving forward, support for new base editors will be added to the application, as they are developed. For the assessment of off-targets generated by the mismatch tolerance of the sgRNA we recommend using Cas-OFFinder (http://www.rgenome.net/ cas-offinder/) [36]. The guide sequences from the output table can be easily transferred and validated, which is especially important for users working on off-target sensitive applications.

Since the 'pegRNA Score' represents a first attempt to rank pegRNA activity and is based on early PE applications [16], it should be only used as an indicator. This score will be refined as dependencies on efficient pegRNA design emerge. Moreover, users are advised to test the efficiency of multiple pegRNAs in their experimental models. This is made easy by PnB Designer. Our dataset of pre-designed PE guides for the majority of ClinVar variants using a wide variety of RTT lengths (Additional files), provides an easy reference for experimenters seeking to model a potentially pathogenic human mutation. Increasing the RTT length even further could increase the number of targetable variants, but this leads to a trade-off of extremely long pegRNAs. Finally, we can say that $\mathrm{PnB}$ Designer is an innovative web application for gene editing research and could support the rapid development of gene editing strategies for translational research.

\section{Availability and requirement}

Project name: PnB Designer

Project home page: http://fgcz-shiny.uzh.ch/PnBDesigner/

GitHub repository: https://github.com/SebastianSiegner/PnB-Designer

Programming language: $R$

Other requirements: No requirements

License: No license needed

Any restrictions to use by non-academics: No access restrictions

\section{Supplementary Information}

The online version contains supplementary material available at https://doi.org/10.1186/s12859-021-04034-6.

Additional file 1. Fig. 1. Example output tables for good and suboptimal guide design for PE and BE. a Example output table for prime editing, showing pegRNA design with a high 'pegRNA Score' and no additional penalties (row 1), as well as pegRNAs suffering from design flaws and consequently assigned with a low 'pegRNA Score' (row 5 and 6). b Example output table for base editing. Three different CBEs can target this SNV. However, two of those show bystander bases in the editing window (marked in blue), which could be edited as well (row 1 and 2). Therefore, the use of Target-AID (row 3) should be prioritized for 'clean' editing at this locus. 
Additional file 2. Fig. 2. Scatterplot, showing 'pegRNA Score' vs. editing efficiency of reported pegRNAs. Reported pegRNAs from Anzalone et al. Fig. 4a with their respective average editing efficiency plotted against their pegRNA Score as calculated by PnB Designer. A linear regression was fitted to investigate the relationship between these two variables and a $R^{2}$ value of 0.297 was obtained. Figure was made using MS Excel (2016).

\section{Abbreviations}

ABEs: CRISPR adenine base editors; BEs: CRISPR base editors; Cas9: CRISPR associated 9; CBEs: Cytidine deaminase base editors; CRISPR: Clustered regularly interspaced short palindromic repeats; dCas9: Enzymatically inactive Cas9; DSB: DNA double strand break; gRNA: Guide RNA; HDR: Homology directed repair; indel: Insertion or deletion; nCas9: Cas9 nickase; PAM: Protospacer adjacent motif; PBS: Primer binding site; pegRNA: Prime editing extended guide RNA; PEs: CRISPR prime editors; RTT: Reverse transcriptase template; SNVs: Single nucleotide variants.

\section{Acknowledgements}

We thank the Functional Genomics Center Zurich (FGCZ) and especially Dr. Hubert Rehrauer and Dr. Ge Tan for hosting the web application. We thank the members of the Corn Lab for helpful discussions. We thank all ClinVar submitters for sharing their data.

\section{Authors' contributions}

SMS, EK, ZK, JEC conceived this project. SMS constructed the PnB Designer reported in this study. SMS, EK, JEC wrote the manuscript. ZK, MSS gave their expertise to improve the manuscript. All authors read and approved the final manuscript.

\section{Funding}

J.E.C. was supported by the NOMIS Foundation and the Lotte and Adolf Hotz-Sprenger Stiftung, E.K. was supported by the Fanconi Anemia Research Foundation. Funding for open access charge: NOMIS Foundation and the Lotte and Adolf Hotz-Sprenger Stiftung. The funders had no role in study design, data collection and analysis, decision to publish, or preparation of the manuscript.

\section{Availability of data and materials}

The dataset(s) supporting the conclusions of this article is(are) included within the article (and its additional file(s)).

\section{Ethics approval and consent to participate}

Not applicable.

\section{Consent for publication}

Not applicable.

\section{Competing interests}

The authors declare that they have no competing interests.

\section{Author details}

${ }^{1}$ Department of Biology, ETH Zurich, Zurich, Switzerland. ${ }^{2}$ Genome Engineering and Measurement Lab, ETH Zurich, Zurich, Switzerland.

Received: 13 May 2020 Accepted: 16 February 2021

Published online: 02 March 2021

\section{References}

1. Knott GJ, Doudna JA. CRISPR-Cas guides the future of genetic engineering. Science. 2018;361(6405):866.

2. Adli M. The CRISPR tool kit for genome editing and beyond. Nat Commun. 2018;9(1):1911.

3. Yeh CD, Richardson CD, Corn JE. Advances in genome editing through control of DNA repair pathways. Nat Cell Biol. 2019;21(12):1468-78.

4. Komor AC, Kim YB, Packer MS, Zuris JA, Liu DR. Programmable editing of a target base in genomic DNA without double-stranded DNA cleavage. Nature. 2016;533(7603):420-4.

5. Gaudelli NM, Komor AC, Rees HA, Packer MS, Badran AH, Bryson DI, Liu DR. Programmable base editing of A•T to G.C in genomic DNA without DNA cleavage. Nature. 2017;551(7681):464-71.

6. Zhang Y, Qin W, Lu X, Xu J, Huang H, Bai H, Li S, Lin S. Programmable base editing of zebrafish genome using a modified CRISPR-Cas9 system. Nat Commun. 2017:8(1):118.

7. Villiger L, Grisch-Chan HM, Lindsay H, Ringnalda F, Pogliano CB, Allegri G, Fingerhut R, Häberle J, Matos J, Robinson MD, et al. Treatment of a metabolic liver disease by in vivo genome base editing in adult mice. Nat Med. 2018;24(10):1519-25.

8. Rees HA, Liu DR. Base editing: precision chemistry on the genome and transcriptome of living cells. Nat Rev Genet. 2018;19(12):770-88.

9. Hao L, Ruiying Q, Xiaoshuang L, Shengxiang L, Rongfang X, Jianbo Y, Pengcheng W. CRISPR/Cas9-mediated adenine base editing in rice genome. Rice Sci. 2019;26(2):125-8.

10. Li C, Zong Y, Wang Y, Jin S, Zhang D, Song Q, Zhang R, Gao C. Expanded base editing in rice and wheat using a Cas9adenosine deaminase fusion. Genome Biol. 2018;19(1):59.

11. Zong Y, Wang Y, Li C, Zhang R, Chen K, Ran Y, Qiu J-L, Wang D, Gao C. Precise base editing in rice, wheat and maize with a Cas9-cytidine deaminase fusion. Nat Biotechnol. 2017;35(5):438-40.

12. Zuo E, Sun Y, Wei W, Yuan T, Ying W, Sun H, Yuan L, Steinmetz LM, Li Y, Yang H. Cytosine base editor generates substantial off-target single-nucleotide variants in mouse embryos. Science. 2019;364(6437):289-92. 
13. Grünewald J, Zhou R, Garcia SP, lyer S, Lareau CA, Aryee MJ, Joung JK. Transcriptome-wide off-target RNA editing induced by CRISPR-guided DNA base editors. Nature. 2019;569(7756):433-7.

14. Grünewald J, Zhou R, lyer S, Lareau CA, Garcia SP, Aryee MJ, Joung JK. CRISPR DNA base editors with reduced RNA off-target and self-editing activities. Nat Biotechnol. 2019;37(9):1041-8.

15. Zhou C, Sun Y, Yan R, Liu Y, Zuo E, Gu C, Han L, Wei Y, Hu X, Zeng R, et al. Off-target RNA mutation induced by DNA base editing and its elimination by mutagenesis. Nature. 2019;571(7764):275-8.

16. Anzalone AV, Randolph PB, Davis JR, Sousa AA, Koblan LW, Levy JM, Chen PJ, Wilson C, Newby GA, Raguram A, et al. Search-and-replace genome editing without double-strand breaks or donor DNA. Nature. 2019;576(7785):149-57.

17. Aida T, Wilde JJ, Yang L, Hou Y, Li M, Xu D, Lin J, Qi P, Lu Z, Feng G. Prime editing primarily induces undesired outcomes in mice. bioRxiv 2020:2020.2008.2006.239723.

18. Rabinowitz R, Abadi S, Almog S, Offen D. Prediction of synonymous corrections by the BE-FF computational tool expands the targeting scope of base editing. Nucleic Acids Res. 2020;48:W340-7.

19. Dandage R, Després PC, Yachie N, Landry CR. A computational workflow for designing libraries of guide RNAs for CRISPR-mediated base editing. Genetics. 2019;212(2):377.

20. Hwang G-H, Park J, Lim K, Kim S, Yu J, Yu E, Kim S-T, Eils R, Kim J-S, Bae S. Web-based design and analysis tools for CRISPR base editing. BMC Bioinform. 2018;19(1):542.

21. Richter MF, Zhao KT, Eton E, Lapinaite A, Newby GA, Thuronyi BW, Wilson C, Koblan LW, Zeng J, Bauer DE, et al. Phage-assisted evolution of an adenine base editor with improved Cas domain compatibility and activity. Nat Biotechnol. 2020;38:883-91.

22. Koblan LW, Doman JL, Wilson C, Levy JM, Tay T, Newby GA, Maianti JP, Raguram A, Liu DR. Improving cytidine and adenine base editors by expression optimization and ancestral reconstruction. Nat Biotechnol. 2018;36(9):843-6.

23. shiny: Easy web applications in R. http://shiny.rstudio.com

24. Gentleman RC, Carey VJ, Bates DM, Bolstad B, Dettling M, Dudoit S, Ellis B, Gautier L, Ge Y, Gentry J, et al. Bioconductor: open software development for computational biology and bioinformatics. Genome Biol. 2004;5(10):R80.

25. Huber W, Carey VJ, Gentleman R, Anders S, Carlson M, Carvalho BS, Bravo HC, Davis S, Gatto L, Girke T, et al. Orchestrating high-throughput genomic analysis with Bioconductor. Nat Methods. 2015;12(2):115-21.

26. Lin Q, Zong Y, Xue C, Wang S, Jin S, Zhu Z, Wang Y, Anzalone AV, Raguram A, Doman JL, et al. Prime genome editing in rice and wheat. Nat Biotechnol. 2020;38:582-5.

27. Tang X, Sretenovic S, Ren Q, Jia X, Li M, Fan T, Yin D, Xiang S, Guo Y, Liu L. Plant prime editors enable precise gene editing in rice cells. Mol Plant. 2020;13:667-70.

28. Gao Z, Herrera-Carrillo E, Berkhout B. Delineation of the exact transcription termination signal for type 3 polymerase III. Mol Ther Nucleic Acids. 2018;10:36-44.

29. Kim HK, Yu G, Park J, Min S, Lee S, Yoon S, Kim HH. Predicting the efficiency of prime editing guide RNAs in human cells. Nat Biotechnol. 2020;39:198-206.

30. Landrum MJ, Lee JM, Riley GR, Jang W, Rubinstein WS, Church DM, Maglott DR. ClinVar: public archive of relationships among sequence variation and human phenotype. Nucleic Acids Res. 2014;42(Database issue):D980-5.

31. Walton RT, Christie KA, Whittaker MN, Kleinstiver BP. Unconstrained genome targeting with near-PAMless engineered CRISPR-Cas9 variants. Science. 2020;368(6488):290.

32. Kleinstiver BP, Prew MS, Tsai SQ, Nguyen NT, Topkar WV, Zheng Z, Joung JK. Broadening the targeting range of Staphylococcus aureus CRISPR-Cas9 by modifying PAM recognition. Nat Biotechnol. 2015;33(12):1293-8.

33. Kleinstiver BP, Prew MS, Tsai SQ, Topkar VV, Nguyen NT, Zheng Z, Gonzales APW, Li Z, Peterson RT, Yeh J-RJ, et al. Engineered CRISPR-Cas9 nucleases with altered PAM specificities. Nature. 2015;523(7561):481-5.

34. Nishimasu H, Shi X, Ishiguro S, Gao L, Hirano S, Okazaki S, Noda T, Abudayyeh OO, Gootenberg JS, Mori H, et al. Engineered CRISPR-Cas9 nuclease with expanded targeting space. Science. 2018;361(6408):1259.

35. Huang TP, Zhao KT, Miller SM, Gaudelli NM, Oakes BL, Fellmann C, Savage DF, Liu DR. Circularly permuted and PAMmodified Cas9 variants broaden the targeting scope of base editors. Nat Biotechnol. 2019:37(6):626-31.

36. Bae S, Park J, Kim J-S. Cas-OFFinder: a fast and versatile algorithm that searches for potential off-target sites of Cas9 RNA-guided endonucleases. Bioinformatics. 2014;30(10):1473-5.

37. Wickham H. ggplot2. WIREs Comput Stat. 2011;3(2):180-5.

38. Chen C-L, Rodiger J, Chung V, Viswanatha R, Mohr SE, Hu Y, Perrimon N. SNP-CRISPR: a web tool for SNP-specific genome editing. G3 (Bethesda). 2020;10(2):489-94.

\section{Publisher's Note}

Springer Nature remains neutral with regard to jurisdictional claims in published maps and institutional affiliations. 